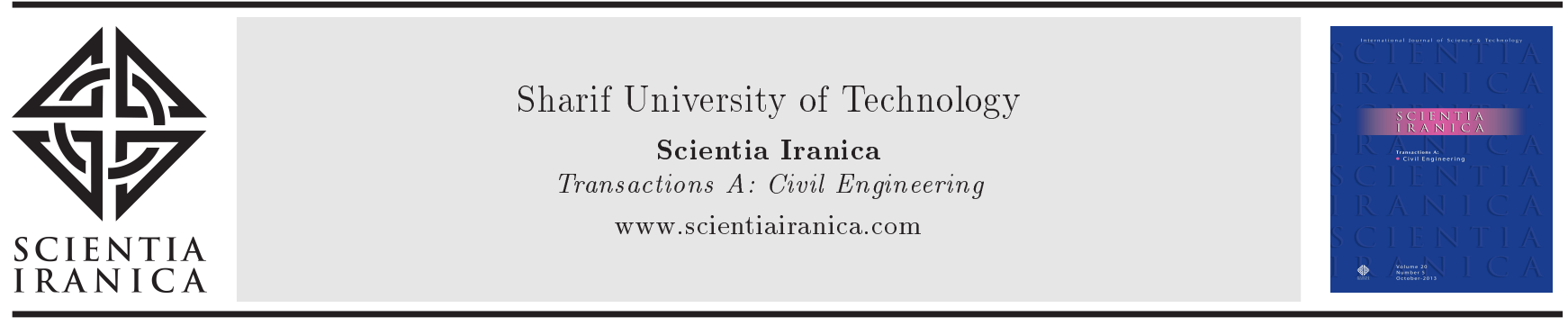

\title{
A comparison of five different models in predicting the shear stress distribution in straight compound channels
}

\author{
Z. Sheikh Khozani* and H. Bonakdari \\ Department of Civil Engineering, Razi University, Kermanshah, Iran.
}

Received 7 April 2015; received in revised form 9 September 2015; accepted 5 October 2015

\section{KEYWORDS}

Compound channel;

Floodplain;

Main channel;

Shear stress

distribution;

Traditional models;

Wetted perimeter.

\begin{abstract}
The ability of five different methods to estimate the shear stress distribution in compound channels is investigated. Methods proposed by Yang and Lim (YLM), Khodasheans and Paquier (KPM), Sterling and Knight (SKM), Zarrati et al. (ZAM), and Bonakdari et al. (BAM) are compared with experimental data. YLM and KPM did not provide reliable results as they produced higher Mean Absolute Percentage of Error (MAPE) values of $25-55 \%$. SKM performed adequately in predicting the pattern of shear stress distribution on the main channel bed, but on a floodplain bed; it predicted a constant value over the entire wetted perimeter. The SKM method outperformed YLM and KPM with 2 to $20 \%$ MAPE. The ZAM and BAM methods produced the best results for shear stress distribution in compound channels with average MAPE of 2.67 and $5.66 \%$ MAPE, respectively. Although ZAM showed more accurate results than BAM, however, BAM required solving much fewer equations than ZAM and presented more accurate results than other geometric methods. Among all models, BAM is proposed as a simple and accurate model for predicting the shear stress distribution in compound channels.
\end{abstract}

(C) 2016 Sharif University of Technology. All rights reserved.

\section{Introduction}

Most of the time, natural river cross sections are found in compound channels, and studying these sections is of specific significance in river engineering and flood risk management. Important concerns in river hydraulics include sedimentation and the transport of solids within these types of cross sections. Shear stress is one of the important problems in sediment transport processes. In many cases, boundary shear stress on the channel wall is predicted in the form of an average value shear stress on the channel wall and bed [1-3]. For many years, a large number of experimental investigations have been carried out in open channels. Studies have shown that it is difficult to

*. Corresponding author. Fax: +98 3137717221

E-mail addresses: y.sheikh2131@gmail.com (Z. Sheikh

Khozani); bonakdari@yahoo.com (H. Bonakdari) determine the boundary shear stress distribution in an open channel [4-9]. To overcome this difficulty, empirical, analytical, and simplified computational methods have been conducted by several researchers [10-21]. Still, even with sophisticated turbulence models, it is challenging to accurately calculate local shear stress values.

Because channel geometry has an important role in variations of boundary shear stress, several theoretical studies have been developed based this concept. Khodashenas and Paquier [11] employed a geometrical method to compute the shear stress distribution by measuring the depth perpendicular to the wall (KPM). In this method, the impact of secondary flow structures and the transfer of momentum between the main channel and its floodplains are neglected. The Shannon entropy concept has been applied by several researchers to predict the shear stress distribution in open channels [12,21-23]. Sterling and Knight [12] 
developed a new approach to predict the distribution of boundary shear stress in open channels (SKM) based on Shannon entropy. Although their proposed method has limitations in reflecting the hydraulic behaviour of open channels, the results indicated that the method could estimate shear stress distribution reasonably well, but it must be developed for practical applications. Yang and Lim [10,14] suggested the transport of surplus energy over the shortest relative distance towards the wall within steady, uniform, and fully developed turbulent flow. They [15] defined a relative distance as the minimum geometrical distance ratio for the energy dissipation capacity of the boundaries (YLM). Based on this concept, the authors proposed an analytical equation for local and mean boundary shear stress along the wetted perimeter. In their model, the impact of secondary current on shear stress was not taken into account either. The semi-analytical equations proposed by Zarrati et al. [24] were aimed to predict shear stress distribution in simple and compound channels (ZAM). These equations were obtained by simplifying a stream-wise vorticity equation and considering secondary Reynolds stresses. The model proposed by Zarrati et al. [24] considered the effect of secondary flows, but contained many equations to estimate shear stress distribution, which is timeconsuming and requires high precision. Bonakdari et al. [19] estimated the shear stress distribution in different channel cross sections using the Tsallis entropy concept and maximized it with the help of Lagrange coefficients (BAM). However, the Tsallis method necessitates solving two explicit equations in order to find the coefficient values.

In this research, the ability of five different methods, namely, KPM, SKM, YLM, ZAM, and BAM in estimating shear stress distribution in compound channels is investigated. The cross section of compound channel is illustrated in Figure 1. All these geometrical models are also compared with each other and the most appropriate model in predicting shear stress is introduced. To verify the results, the laboratorial outcomes obtained by Rajaratnam and Ahmadi [25], Myers and Elsawy [26], and Cokljat and Younis [27] are utilized.

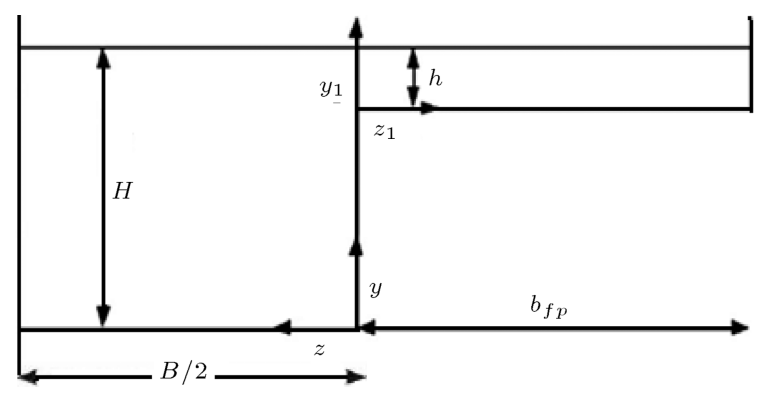

Figure 1. Compound channel transverse section characteristics.

\section{Review of methods}

Five methods for estimating the shear stress distribution in a compound open channel are compared with each other against experimental data. These methods were selected because they are sufficiently general to predict the shear stress distribution, and they are simple enough for engineering applications.

\subsection{Yang and Lim's Method (YLM)}

Yang and Lim [10,14] defined a relative distance as the minimum geometrical distance ratio for the energy dissipation capacity of boundaries. For smooth boundaries, this relative distance represents the dissipation capacity of a boundary with a viscous length scale as $\frac{v}{u_{*}}$ (where $v$ is the kinematic viscosity and $u_{*}$ is the shear velocity), and for rough boundaries, this characteristic length is scaled according to the roughness height. To accomplish this, boundaries are divided according to the transverse section shape.

For a wide channel $\left(\frac{b}{h}>2\right)$, the intersection of division lines is located above the free surface, and the shear stress distributions of the bed and wall in compound channels (Figure 1) are written according to El Kadi Abderrezzak [16]:

$$
\begin{aligned}
& \tau_{b}(z)=\rho g z S \xi, \quad 0<z \leq \frac{H-h}{\xi}, \\
& \tau_{b}(z)=\rho g(H-h) S, \quad \frac{H-h}{\xi}<z \leq\left(B-\frac{H-h}{\xi}\right) \\
& \tau_{b}(z)=\rho g \xi(B-z) S, \quad\left(B-\frac{H-h}{\xi}\right)<z \leq B, \quad(3) \\
& \tau_{w}(y)=\rho g y S, \quad 0<y \leq(H-h), \\
& \tau_{b}\left(z_{1}\right)=\rho g h S, \quad 0<z_{1} \leq\left(b_{f p}-\frac{h}{\xi}\right), \\
& \tau_{b}\left(z_{1}\right)=\rho g\left(b_{f p}-z_{1}\right) S \xi, \quad\left(b_{f p}-\frac{h}{\xi}\right)<z_{1} \leq b_{f p},(6) \\
& \tau_{w}\left(y_{1}\right)=\rho g y_{1} S, \quad 0<y_{1} \leq h .
\end{aligned}
$$

Parameter $\xi$ in the above equations is calculated as:

$$
-2 \xi^{3}+\frac{2 H}{B} \xi^{2}+1=0
$$

where $\rho$ is the fluid density, $g$ is the gravitational acceleration, $S$ is the energy slope, $b$ is the channel width, and $h$ is the water depth. For narrow channels $\left(\frac{b}{h} \leq 2\right)$ in which line intersections are below the free surface and considering the experimental data used in the present study relates to wide channel sections, explaining shear stress equations for narrow channel sections is avoided. 


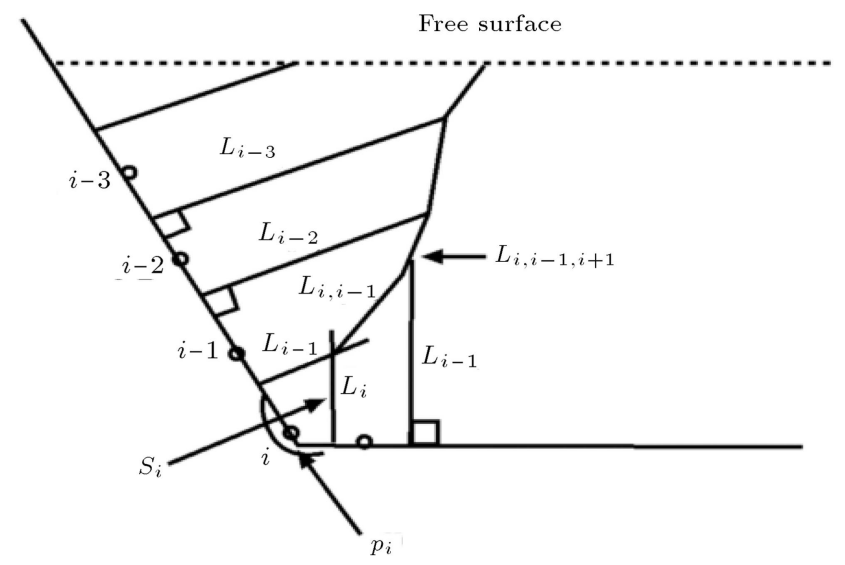

Figure 2. Schematic illustration of the areas determined by KPM.

\subsection{Khodashenas and Paquier's Method ( $K P M)$}

The normal area method is one of the techniques of estimating shear stress distribution. With this method, the boundary shear stress is estimated from the area between two drowned normal to bed of channel. This method fails when channel walls have a steep slope, because the intersection of normal occurs below the water surface. To solve this problem, Khodashenas and Paquier [11] extended the normal area method and predicted shear stress distribution in an irregular cross section of a channel. The shear stress distribution was calculated as follows:

1. The wetted perimeter is divided into small segments (Figure 2).

2. The mediator of each segment is drawn.

3. When each mediator intersects the normal line, they should merge with each other. The direction of the new line of order 2 created is computed as:

$$
\hat{L}_{i, i-1}=\frac{1}{2}\left(\hat{L}_{i}+\hat{L}_{i-1}\right) .
$$

4. When this new line intersects the normal line or other high-order lines, its angle is computed with the weighted mean of the previous line. This procedure continues until the water surface is reached:

$$
\hat{L}_{i, i-1, i+1}=\frac{1}{3}\left(2 \hat{L}_{i, i-1}+\hat{L}_{i+1}\right) .
$$

5. The area between the final lines $\left(S_{i}\right)$ is calculated and the shear stress is estimated by:

$$
\tau_{i}=\rho g R_{h i} S,
$$

where $R_{h i}=S_{i} / P_{i}$ is the local hydraulic radius, $g$ is the gravity acceleration, $\rho$ is the water density, and $S$ is the energy slope.

\subsection{Sterling and Knight's Method (SKM)}

Sterling and Knight [12] used the Lagrange coefficient to maximize the Shannon entropy and introduce an equation to predict shear stress. Based on this method, the shear stress distribution in a compound channel with the section shown in Figure 1 is predicted as:

$$
\begin{aligned}
\tau_{b}(z) & =\frac{1}{\lambda_{b m}} \ln \left[1+\left(e^{\lambda_{b m} \tau_{\max }(b m)}-1\right) \frac{2\left(z-z_{c}\right)}{B}\right] \\
z_{c} & <z<\frac{B}{2}, \\
\tau_{w}(y) & =\frac{1}{\lambda_{w m}} \ln \left[1+\left(e^{\lambda_{w m} \tau_{\max }(w m)}-1\right) \frac{2\left(y-y_{c}\right)}{H-h}\right] \\
y_{c} & <y<H-h, \\
\tau_{b}\left(z_{1}\right) & =\frac{1}{\lambda_{b f p}} \ln \left[1+\left(e^{\lambda_{b f p} \tau_{\max }(b f p)}-1\right)\right] \\
0 & <z_{1}<b_{f p}, \\
\tau_{w}\left(y_{1}\right) & =\frac{1}{\lambda_{w f p}} \ln \left(1+\left(e^{\lambda_{w f p} \tau_{\max }(w f p)}-1\right) \frac{\left(y_{1}-y_{c}\right)}{h}\right) \\
y_{c} & <y_{1}<h,
\end{aligned}
$$

where $\tau_{w}(y)$ and $\tau_{w}\left(y_{1}\right)$ are shear stress at the wall of the main channel and floodplain, respectively; $\tau_{b}(z)$ and $\tau_{b}\left(z_{1}\right)$ are the shear stress on the bed of the main channel and floodplain, respectively; $\tau_{\max (w f p)}$, $\tau_{\max (b f p)}, \tau_{\max (w m)}$, and $\tau_{\max (b m)}$ are the maximum shear stress at the wall and bed of the floodplain and main channel; and $y_{c}$ and $z_{c}$ are constant values of $5 \mathrm{~mm}$ [12]. Eqs. (12) to (15) are used to predict the shear stress distribution in sub sections of the compound channel shown in Figure 1. In Eqs. (12) to (15), the $\lambda$ parameter is calculated as follows:

$$
\lambda=\left(\frac{\tau_{\max } e^{\lambda \tau_{\max }}}{e^{\lambda \tau_{\max }}-1}-\rho g R S\right)^{-1} .
$$

In order to use Eqs. (12) to (15) as well as (16), the mean and maximum shear stress should be calculated earlier. Therefore, the relations presented by Knight et al. [1] are applied as follows:

$$
\begin{aligned}
& \frac{\tau_{\max (w)}}{\rho g R S}=0.01 \% S F_{w}\left(1+P_{b} / P_{w}\right) \\
& \frac{\tau_{\operatorname{mean}(b)}}{\rho g R S}=\left(1-0.01 \% S F_{w}\right)\left(1+\frac{1}{P_{b} / P_{w}}\right) \\
& \frac{\tau_{\max (w)}}{\rho g R S}=0.01 \% S F_{w}\left[2.0372\left(P_{b} / P_{w}\right)^{0.7108}\right] \\
& \frac{\tau_{\max (b)}}{\rho g R S}=\left(1-0.01 \% S F_{w}\right)\left[2.1697\left(P_{b} / P_{w}\right)^{-0.3287}\right]
\end{aligned}
$$


where $\tau_{\text {mean }(w)}$ and $\tau_{\text {mean }(b)}$ are the mean shear stress at the wall and bed, respectively; $\rho$ is the fluid density; $g$ is gravitational acceleration; $R$ is the hydraulic radius; $S$ is the bed slope; $P_{b}$ and $P_{w}$ are the wetted perimeter corresponding to the bed and wall of the channel, respectively; $\tau_{\max (w)}$ and $\tau_{\max (b)}$ are the maximum shear stress at the wall and bed, respectively; and $\% \mathrm{SF}_{w}$ is the percentage of shear force carried by walls and is evaluated as follows:

$$
\% S F_{w}=C_{s f} \exp \left(-3.23 \log \left(P_{b} / C^{\prime} P_{w}+1\right)+4.6052\right),
$$

where $C_{s f}=1$ for $P_{b} / P_{w}<4.374$, unless $C_{s f}=$ $0.6603\left(P_{b} / P_{w}\right)^{0.28125}$ and in subcritical flow $C^{\prime}=1.38$. Thereby, for a given channel, depending on the water depth and bed slope, the transverse distribution of shear stress can be estimated.

\subsection{Zarrati et al.'s Method (ZAM)}

An analytical model was presented by Zarrati et al. [24] to predict the shear stress distribution in channels with rectangular, trapezoidal, and compound cross sections. They derived semi-analytical equations based on a simplified streamwise vorticity equation including secondary Reynolds stresses. In this method, the effect of additional secondary flows is considered, which is due to the shear layer between the main channel and the floodplain. To compute the shear stress on the wetted perimeter of a compound channel, the channel cross section was divided into regions, as shown in Figure 3.

Two equations for predicting the shear stress along the bed of a main channel are:

$$
\begin{aligned}
& \frac{\tau_{b}(z)}{\tau_{\text {mean }(b m)}}=A_{1} \frac{\frac{2 z}{B}}{\frac{2 z}{B}+\frac{2 a}{B} \delta_{1}}+C_{1} \frac{2 z}{B} \quad 0<z<a, \\
& \frac{\tau_{b}(z)}{\tau_{\text {mean }(b m)}}=A_{2} \frac{2 z}{B}+C_{2}, \quad a<z<\frac{B}{2},
\end{aligned}
$$

where $\delta_{1}=0.02, a=H-h-z_{0}$, and $z_{0}=$ $0.026827\left(e^{(-2.812148(h / H-1))}-1\right) b_{f p}$.

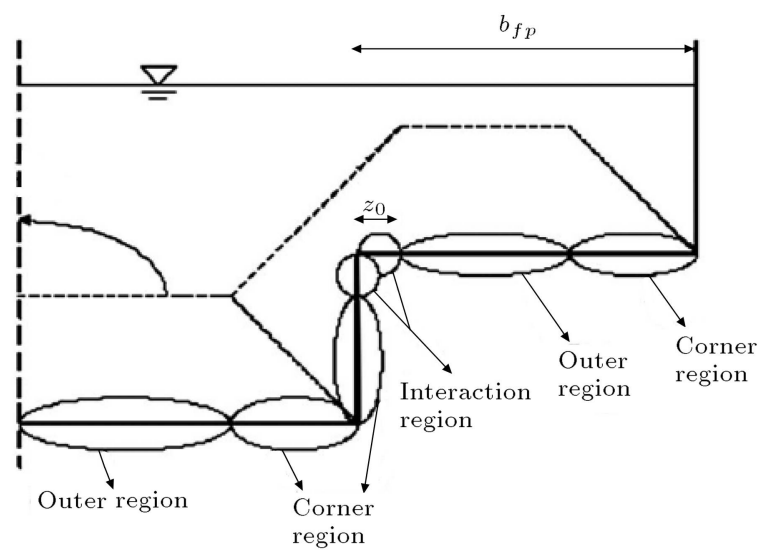

Figure 3. Geometric parameters of a compound channel [24].
To predict the shear stress on the main channel wall, the following equations are proposed:

$$
\begin{gathered}
\frac{\tau_{w}(y)}{\tau_{\text {mean }(w m)}}=A_{2}^{\prime} \frac{\frac{y}{H-h}}{\frac{y}{H-h}+\frac{H-h-z_{0}}{H-h} \delta_{1}}+C_{2}^{\prime} \frac{y}{H-h} \\
0<y<H-h-z_{0}, \\
\frac{\tau_{w}(y)}{\tau_{\text {mean }(w m)}}=A_{1}^{\prime}\left(\frac{H-h-y}{z_{0}}\right)^{2}+B_{1}^{\prime}\left(\frac{H-h-y}{z_{0}}\right)+C_{1}^{\prime} \\
H-h-z_{0}<y<H-h .
\end{gathered}
$$

To calculate the shear stress distribution along a floodplain bed, Eqs. (26) to (28) are used:

$$
\begin{aligned}
\frac{\tau_{b}\left(z_{1}\right)}{\tau_{\text {mean }(b f p)}}=A_{1}\left(\frac{z_{1}}{z_{0}}\right)^{2}+B_{1}\left(\frac{z_{1}}{z_{0}}\right)+C_{1} \\
0<z_{1}<z_{0} \\
\frac{\tau_{b}\left(z_{1}\right)}{\tau_{\text {mean }(b f p)}}=A_{2}\left(\frac{z_{1}}{z_{0}}\right)^{2}+B_{2}, \quad z_{0}<z_{1}<b_{f p}-a,(27) \\
\frac{\tau_{b}\left(z_{1}\right)}{\tau_{\text {mean }(b f p)}}=A_{3} \frac{-\frac{z_{1}-b_{f p}}{b_{f p}}}{-\frac{z_{1}-b_{f p}}{b_{f p}}+0.02 \frac{a}{b_{f p}}}-B_{3} \frac{z_{1}-b_{f p}}{b_{f p}} \\
b_{f p}-a<z_{1}<b_{f p} .
\end{aligned}
$$

Ultimately, the lateral stress distribution on the floodplain wall can be calculated with Eqs. (29) and (30):

$$
\begin{aligned}
\frac{\tau_{w}\left(y_{1}\right)}{\tau_{\text {mean }(w f p)}} & =A_{1}^{\prime} \frac{\frac{y_{1}}{h}}{\frac{y_{1}}{h}+\frac{a}{h} \delta_{1}}+C_{1}^{\prime} \frac{y_{1}}{h} \\
0<y_{1} & <a \\
\frac{\tau_{w}\left(y_{1}\right)}{\tau_{\text {mean }(w f p)}} & =A_{2}^{\prime} \frac{1-\frac{y_{1}}{h}}{1-\frac{y_{1}}{h}+\frac{h-a}{h} \delta_{2}}+C_{2}^{\prime}\left(1-\frac{y_{1}}{h}\right), \\
0<y_{1} & <h
\end{aligned}
$$

In these equations, $a$ is the width of the corner region; $z_{0}$ is the interaction zone width; $\delta_{2}=0.02 ; A_{1}, B_{1}$, $C_{1}, A_{2}, B_{2}, C_{2}, A_{3}, B_{3}$, and $C_{3}$, are bed shear stress distribution coefficients; and $A_{1}^{\prime}, B_{1}^{\prime}, C_{1}^{\prime}, A_{2}^{\prime}$, $B_{2}^{\prime}$ represent wall shear stress distribution. These coefficients can be calculated with some equations mentioned in the Zarrati et al. [24].

\subsection{Bonakdari et al.'s method (BAM)}

Bonakdari et al. [19] employed the Tsallis entropy to derive the shear stress distribution in different channel cross sections based on two simple constraints: 
the total probability, and (2) conservation of mass, along with maximizing the entropy function using Lagrange coefficients. The parameters of the derived shear stress distribution were determined using these two constraints. They studied the model's accuracy in circular, circular with flat bed, rectangular, and compound cross sections. Only one cross section of a compound channels was investigated in the study.

Equations were derived for estimating the shear stress distribution in sub-sections of a compound channel as follows:

$$
\begin{aligned}
\tau_{b}(z) & =\frac{1}{\lambda_{b m}}\left(\left(\lambda_{b m}^{\prime}\right)^{k}+\frac{\lambda_{b m} z}{B / 2}\right)^{1 / k}-\frac{\lambda_{b m}^{\prime}}{\lambda_{b m}} \\
z_{c} & \leq z \leq \frac{B}{2}, \\
\tau_{w}(y) & =\frac{1}{\lambda_{w m}}\left(\left(\lambda_{w m}^{\prime}{ }^{k}\right)+\frac{\lambda_{w m} y}{H-h}\right)^{1 / k}-\frac{\lambda_{w m}^{\prime}}{\lambda_{w m}} \\
y_{c} & \leq y \leq H-h .
\end{aligned}
$$

For a floodplain, the Divided Channel Method (DCM) was employed, and the following equations were obtained:

$$
\begin{gathered}
\tau_{b}\left(z_{1}\right)=\frac{1}{\lambda_{b f p}}\left(\left(\lambda_{b f p}^{\prime}\right)^{k}+\frac{\lambda_{b f p}\left(b_{f p}-z_{1}\right)}{b_{f p}}\right)^{1 / k}-\frac{\lambda_{b f p}^{\prime}}{\lambda_{b f p}} \\
0 \leq z_{1} \leq b_{f p}-z_{c}, \\
\tau_{w}\left(y_{1}\right)=\frac{1}{\lambda_{w f p}}\left(\left(\lambda_{w f p}^{\prime}\right)^{k}+\frac{\lambda_{w f p} y}{h}\right)^{1 / k}-\frac{\lambda_{w f p}^{\prime}}{\lambda_{w f p}} \\
y_{c} \leq y_{1} \leq h .
\end{gathered}
$$

To compute the Lagrangian coefficients $\lambda$ and $\lambda^{\prime}$, Eqs. (35) and (36) are used, which are obtained based on two constraints:

$$
\begin{aligned}
& {\left[\lambda^{\prime}+\lambda \tau_{\max }\right]^{k}-\left[\lambda^{\prime}\right]^{k}=\lambda k^{k}} \\
& \frac{\tau_{\max }}{\lambda}\left[\lambda^{\prime}+\lambda \tau_{\max }\right]^{k}-\frac{1}{\lambda^{2}} \frac{1}{k+1}\left[\lambda^{\prime}+\lambda \tau_{\max }\right]^{k+1} \\
& \quad+\frac{1}{\lambda^{2}} \frac{1}{k+1}\left[\lambda^{\prime}\right]^{k+1}=k^{k} \tau_{\text {mean }}
\end{aligned}
$$

where $\tau_{\text {max }}$ and $\tau_{\text {mean }}$ are the maximum and mean shear stress values on the wall and bed of the channel. In order to use Eqs. (35) and (36), the average and maximum shear stresses should be previously calculated using the relations presented by Knight et al. [1] and introduced in Eqs. (17) to (21).

\section{Shear stress distribution in sub-sections of a compound channel}

\subsection{Shear stress on a channel wall in a floodplain}

With the help of YLM, SKM, KPM, ZAM, and BAM, the shear stress distribution on a compound channel wall in a floodplain is calculated for two different heights, $h / H=0.25$ and $h / H=0.4$, and is shown in Figures 4 and 5 . The results are compared with the laboratorial outcomes of Myers and Elsawy [26], where the channel geometry was the same and only the flow depth differed. According to Figures 3 and 4, BAM predicted the most appropriate shear stress distribution results for a floodplain wall compared to other methods. The ZAM method presented smaller error in estimating the shear stress on a floodplain than other methods for $h / H=0.25$. ZAM predicted small values for shear stress distribution in the corner region, but estimated more accurate shear stress distribution results for $\frac{y_{1}}{h}>0.2$. As seen in Figures 4 and 5, if the channel geometry did not change, ZAM predicted

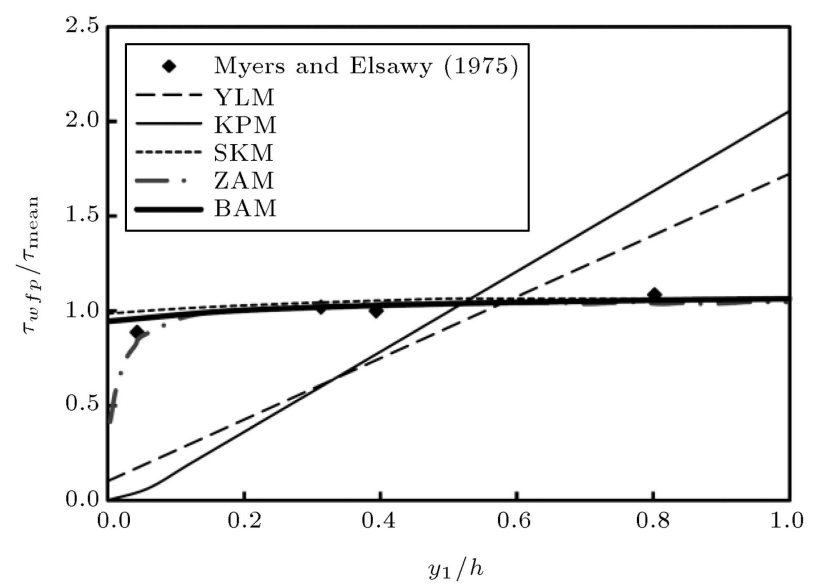

Figure 4. Relative shear stress distribution on a floodplain channel wall $(h / H=0.25)$.

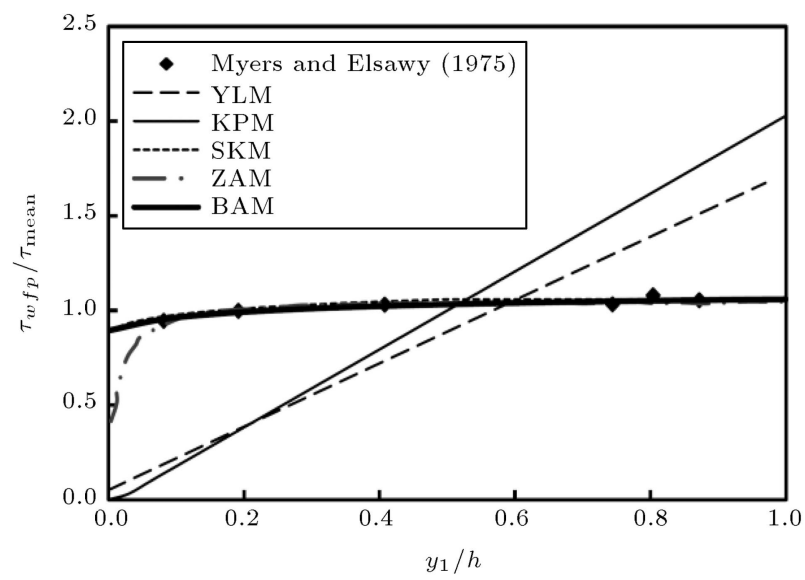

Figure 5. Relative shear stress distribution on a floodplain channel wall $(h / H=0.4)$. 
similar shear stress distribution values. On the other hand, depth variation did not influence the prediction of shear stress on a floodplain wall using Eqs. (29) and (30).

Moreover, the SKM method predicted results compatible with laboratorial outcomes. The SKM predicted similar values for all wetted perimeters of a floodplain wall, but these values were close to the mean of observed values. The YLM and KPM methods did not produce good prediction results, as on one half of the wall, the results were underestimated; and for the other half, the results were overestimated. The shear stress distribution pattern predicted that using YLM, and KPM forms a sloping line where the shear stress values increase as they approach the water-air interface. It can be deducted that the BAM, SKM, and ZAM methods predicted results close to laboratorial values. All three methods produced reasonable results with percentage of error less than $5 \%$.

\subsection{Shear stress on the floodplain bed}

The estimation results for shear stress on the bed of a floodplain are illustrated in Figures 6 and 7 . The bed shear stress values are calculated for two different heights with ratios of $h / H=0.325$ and $h / H=0.5$ with the help of the five methods mentioned in the previous section. The results are compared with the laboratorial outcomes of Rajaratnam and Ahmadi [25] and Myers and Elsawy [26]. According to Figure 6, the KPM model predicted shear stress distribution patterns suitably for both heights and corresponded well with experimental results. However, the model exhibited poor performance for $h / H=0.325$ at the interface of the main channel and floodplain. The YLM method had similar results to KPM, although it introduced higher error. ZAM demonstrated a better shear stress prediction with increasing depth. Since the secondary flow effect was considered in the ZAM model equations, its results were much closer to the exper-

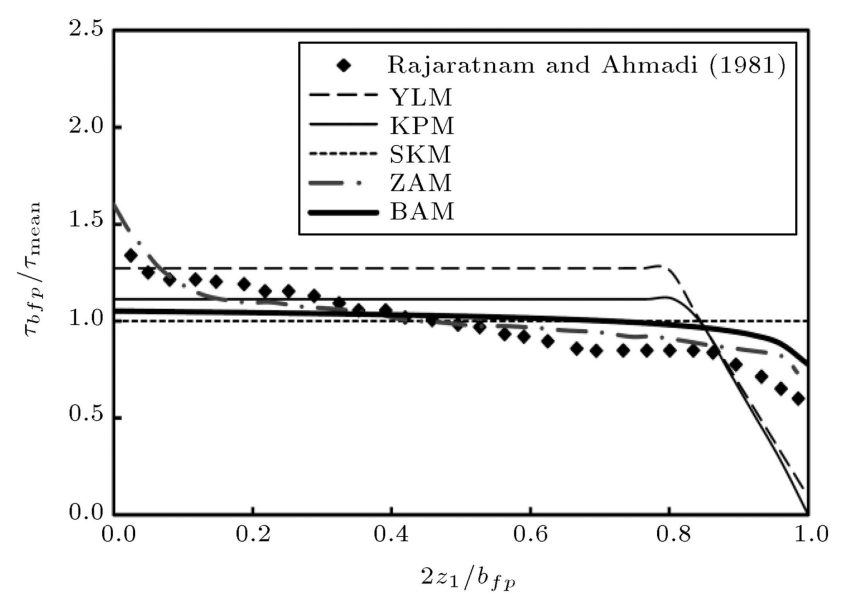

Figure 6. Relative shear stress distribution on floodplain channel bed $(h / H=0.325)$.

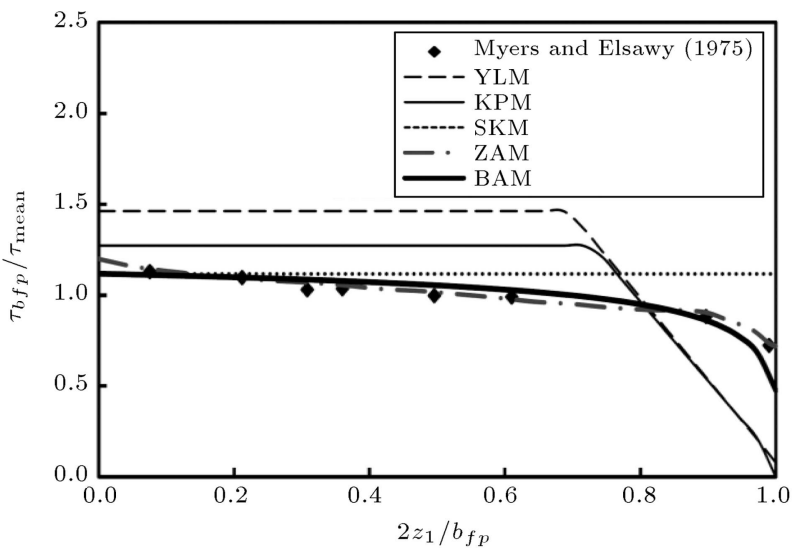

Figure 7. Relative shear stress distribution on floodplain channel bed $(h / H=0.5)$.

imental results compared with the other methods for the beginning part of the wetted perimeter. Generally, because the fifteen equations and parameters employed to estimate the shear stress distribution with the ZAM model, using this method necessitates high accuracy and is time-consuming.

SKM is quite an appropriate relation for shear stress prediction on a floodplain bed. This method predicts greater shear stress than laboratorial values for the channel corners and lower shear stress at the main channel interface with the floodplain. Although, with increment in flow height at the interface of the main channel and the floodplain, the difference between the SKM and laboratorial outcomes decreases. The same as for the channel wall, compared with the other methods, BAM predicts the closest results to laboratorial outcomes for a floodplain, but it predicts underestimated shear stress values for the interface of the main channel and the floodplain. Although, with increasing flow height, the difference between the values predicted by BAM and experimental data decreased somewhat, whereby at $h / H=0.5$, the results were entirely compatible with laboratorial outcomes. BAM had $3.28 \%$ error at $h / H=0.5$, which indicates this method's high capability at this ratio.

\subsection{Shear stress on a main channel wall}

The shear stress distribution on a main channel wall at $h / H=0.333$ in two sections with different dimensions is calculated and illustrated in Figures 8 and 9. The results of the five mentioned methods are compared with the experimental results of Myers and Elsawy [26] and Rajaratnam and Ahmadi [25]. As seen in Figures 8 and 9 , at similar flow depths and different cross section dimensions as well as channel slopes, the prediction of the shear stress values obviously differed among the methods because all mentioned methods are geometrical models. The KPM and YLM presented the same linear distribution that did not match experimental data and contained high errors. It should be mentioned 


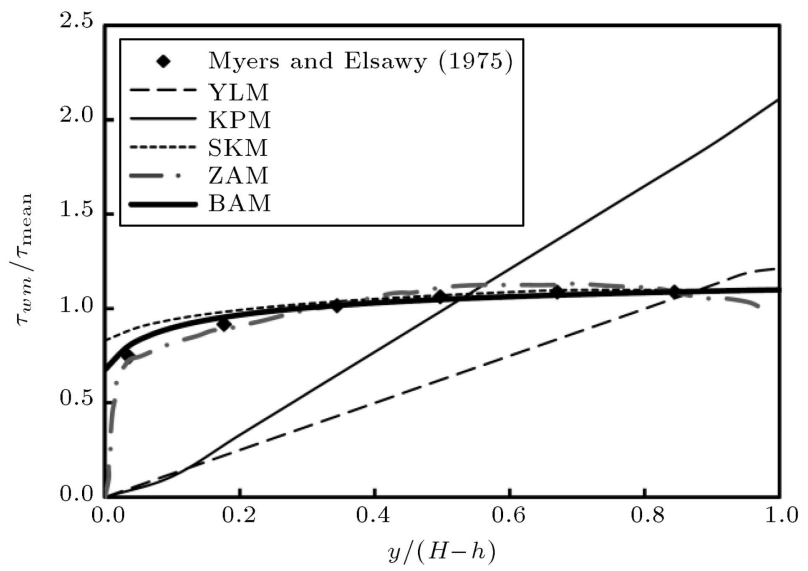

Figure 8. Relative shear stress distribution on a main channel wall $(h / H=0.333)$.

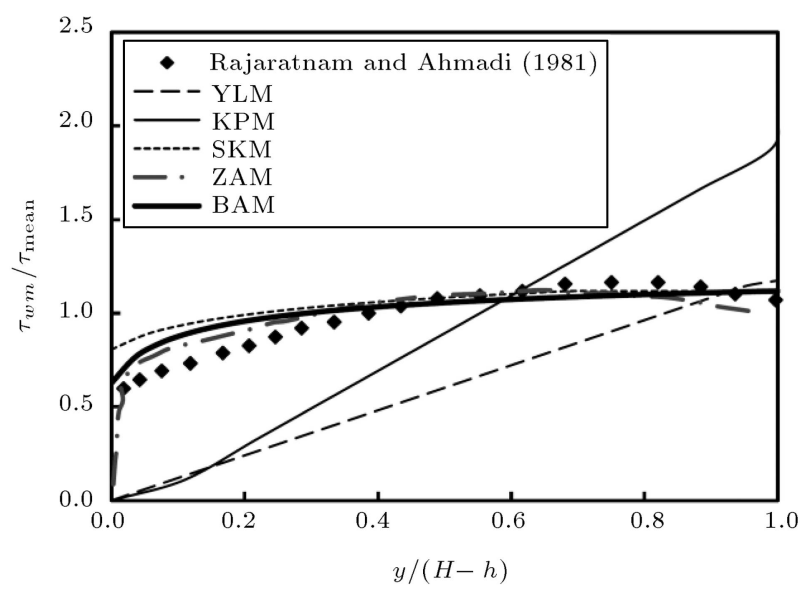

Figure 9. Relative shear stress distribution on a main channel wall $(h / H=0.333)$.

that KPM predicted the worst results among the models. ZAM predicted very small shear stress values at the beginning of the channel. A little further from the separation area, the results were almost equal to experimental data for $0.4<\frac{y}{(H-h)}<0.8$, and the predicted results were somewhat overestimated. This method presented results closer to experimental data compared with the YLM and KPM models.

SKM made acceptable predictions, but it did not match well with experimental results at the beginning of the section. The BAM model made predictions considerably close to experimental data (especially in Figure 8) and made superior estimations for shear stress distribution at the beginning of the section compared with SKM. In addition, BAM made accurate predictions in the flow separation section as well as the interface of the main channel and floodplain. Experimental data of Rajaratnam and Ahmadi [25] are used for various section dimensions in Figure 9. The same as before, the BAM model presented better results than those of the other four mentioned methods.

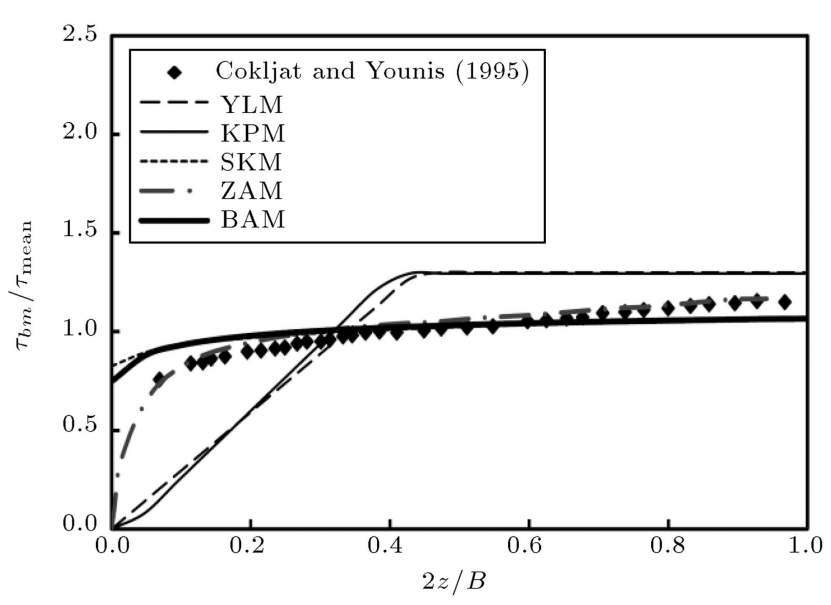

Figure 10. Shear stress distribution on the main channel bed $(h / H=0.5)$.

\subsection{Shear stress on the main channel bed}

The shear stress distribution on the bed of the main channel predicted by the five introduced models is shown in Figure 10 and compared with Cokljat and Younis' experimental data [27]. Figure 10 indicates that the KPM and YLM models predicted a similar shear stress distribution pattern for the main channel bed that is contrary to laboratorial outcomes. The SKM model predicted a uniform distribution for shear stress, which is better than the KPM and YLM models. The ZAM model was more adapted to experimental data. However, the ZAM model has more equations and parameters for calculating the shear stress on the main channel bed compared with the other methods. It is also more time-consuming and needs much more calculation accuracy. The BAM model presented the most appropriate results for predicting the shear stress distribution on the main channel bed using fewer and simpler equations than the ZAM model.

\section{Model performance}

The performance of the YLM, KPM, SKM, ZAM, and BAM models in predicting the shear stress distribution in compound cross sections is evaluated using statistical comparisons of the predicted and observed outputs. The comparison involves four of the most commonly applied error measures: Root Mean Aquare Error (RMSE), Mean Absolute Percentage of Error (MAPE), Mean Square Error (MSE), and BIAS. These statistical parameters are calculated as:

$$
\begin{aligned}
\text { RMSE } & =\sqrt{\frac{\sum_{i=1}^{n}\left(\tau_{i p}-\tau_{i m}\right)^{2}}{n}}, \\
\text { MAPE } & =100 \frac{1}{n} \sum_{i=1}^{n}\left|\frac{\tau_{i p}-\tau_{i m}}{\tau_{i m}}\right|,
\end{aligned}
$$




$$
\begin{aligned}
\mathrm{MSE} & =\frac{\sum_{i=1}^{n}\left(\tau_{i p}-\tau_{i m}\right)^{2}}{n}, \\
\mathrm{BIAS} & =\frac{\sum_{i=1}^{n} \tau_{i p}-\tau_{i m}}{n} .
\end{aligned}
$$

Table 1 displays the comparison results between each model and experimental data. YLM with RMSE of 0.442 outperformed KPM with RMSE of 0.556 in estimating the shear stress distribution on a floodplain wall. SKM, ZAM, and BAM produced similar results to some extent. Among all models, ZAM performed better with $\mathrm{RMSE}=0.045, \mathrm{MAPE}=4.12, \mathrm{MSE}=0.002$, and $\mathrm{BIAS}=-0.017$ (for $h / H=0.25$ ). As seen in Table 1, KPM had lower error values in predicting the shear stress on the bed of main and floodplain channels than YLM. In other words, YLM exhibited higher ability than KPM in predicting the shear stress distribution on a floodplain wall, and for other subsections, KPM outperformed YLM. ZAM predicted the shear stress distribution on a floodplain bed with lower error $(\mathrm{RMSE}=0.073, \mathrm{MAPE}=7.194, \mathrm{MSE}=0.005$, and BIAS=0.0185) than SKM and BAM, whose average RMSE and average MAPE values were 0.134 and 13.09 also 0.096 and 9.17 , respectively. Both methods performed weakly due to the higher error values. The SKM model provided lower statistical parameter values compared with the KPM and YLM models. Considering that secondary flows affect the suggested equations in the ZAM model, ZAM seemed to be more accurate than all other methods. This method could not predict more accurate shear stress values at the interface of the main channel and floodplain, and it predicted the shear stress at the main channel wall with higher error (RMSE of 0.052) compared with BAM and

\begin{tabular}{|c|c|c|c|c|c|c|c|c|}
\hline Experiment & Subsection & $h / H$ & $\begin{array}{l}\text { Statistical } \\
\text { parameters }\end{array}$ & YLM & KPM & SKM & ZAM & BAM \\
\hline \multirow{8}{*}{$\begin{array}{l}\text { Myers and } \\
\text { Elsawy [26] }\end{array}$} & \multirow{8}{*}{$\begin{array}{l}\text { Wall of } \\
\text { floodplain }\end{array}$} & \multirow{4}{*}{0.25} & RMSE & 0.442 & 0.557 & 0.069 & 0.045 & 0.085 \\
\hline & & & MAPE & 41.61 & 51.84 & 5.91 & 4.12 & 5.18 \\
\hline & & & MSE & 0.195 & 0.310 & 0.005 & 0.002 & 0.007 \\
\hline & & & BIAS & -0.24 & -0.207 & 0.046 & -0.017 & 0.043 \\
\hline & & \multirow{4}{*}{0.4} & RMSE & 0.509 & 0.584 & 0.026 & 0.025 & 0.012 \\
\hline & & & MAPE & 46.52 & 54.41 & 2.391 & 1.981 & 0.557 \\
\hline & & & MSE & 0.259 & 0.341 & 0.001 & 0.001 & 0.000 \\
\hline & & & BIAS & -0.13 & 0.013 & -0.014 & -0.012 & -0.003 \\
\hline \multirow{4}{*}{$\begin{array}{c}\text { Rajaratnam and } \\
\text { Ahmadi }[25]\end{array}$} & \multirow{8}{*}{$\begin{array}{c}\text { Bed of } \\
\text { floodplain }\end{array}$} & \multirow{4}{*}{0.5} & RMSE & 0.277 & 0.213 & 0.197 & 0.076 & 0.141 \\
\hline & & & MAPE & 27.39 & 20.54 & 19.12 & 7.12 & 13.62 \\
\hline & & & MSE & 0.077 & 0.045 & 0.039 & 0.006 & 0.02 \\
\hline & & & BIAS & 0.138 & 0.006 & 0.069 & 0.026 & 0.023 \\
\hline \multirow{4}{*}{$\begin{array}{l}\text { Myers and } \\
\text { Elsawy [26] }\end{array}$} & & \multirow{4}{*}{0.325} & RMSE & 0.511 & 0.480 & 0.070 & 0.070 & 0.052 \\
\hline & & & MAPE & 54.28 & 48.79 & 7.055 & 7.267 & 4.713 \\
\hline & & & MSE & 0.261 & 0.231 & 0.005 & 0.004 & 0.003 \\
\hline & & & BIAS & -0.455 & -0.067 & 0.028 & 0.011 & 0.032 \\
\hline \multirow{4}{*}{$\begin{array}{l}\text { Rajaratnam and } \\
\text { Ahmadi [25] }\end{array}$} & \multirow{8}{*}{$\begin{array}{l}\text { Wall of } \\
\text { main channel }\end{array}$} & \multirow{4}{*}{0.333} & RMSE & 0.511 & 0.480 & 0.121 & 0.070 & 0.089 \\
\hline & & & MAPE & 54.28 & 48.79 & 11.73 & 7.267 & 8.503 \\
\hline & & & MSE & 0.261 & 0.231 & 0.015 & 0.004 & 0.008 \\
\hline & & & BIAS & -0.455 & -0.067 & 0.066 & 0.011 & 0.0413 \\
\hline \multirow{4}{*}{$\begin{array}{l}\text { Myers and } \\
\text { Elsawy [26] }\end{array}$} & & \multirow{4}{*}{0.333} & RMSE & 0.505 & 0.516 & 0.091 & 0.036 & 0.025 \\
\hline & & & MAPE & 48.07 & 48.57 & 7.246 & 3.476 & 1.802 \\
\hline & & & MSE & 0.255 & 0.266 & 0.008 & 0.001 & 0.001 \\
\hline & & & BIAS & -0.439 & -0.084 & 0.058 & -0.007 & 0.014 \\
\hline \multirow{4}{*}{$\begin{array}{l}\text { Cokeljat and } \\
\text { Younis [27] }\end{array}$} & \multirow{4}{*}{$\begin{array}{l}\text { Bed of } \\
\text { main channel }\end{array}$} & \multirow{4}{*}{0.5} & RMSE & 0.264 & 0.262 & 0.063 & 0.047 & 0.058 \\
\hline & & & MAPE & 24.11 & 23.78 & 5.516 & 4.456 & 5.266 \\
\hline & & & MSE & 0.070 & 0.068 & 0.004 & 0.002 & 0.003 \\
\hline & & & BIAS & -0.005 & -0.008 & 0.021 & 0.041 & 0.011 \\
\hline
\end{tabular}

Table 1. Comparison of experimental results for a compound channel with the results obtained with different models. 
SKM (RMSE of 0.038 and 0.046 respectively). Note that ZAM includes many dependent equations and parameters to estimate shear stress distribution, which necessitates much more time and higher accuracy. The BAM model performed the best in predicting shear stress distribution with smaller values of MAPE\% (less than $6 \%$ ). Compared to the experimental results of Rajaratnam and Ahmadi [25], the BAM model did not produce acceptable results (RMSE of 0.14 ).

Since flow separation is a parameter with influence on the interface of a main channel and floodplain as well as energy wastage, and the models applied in this study are analytical and geometrical methods; the shear stress values in this region are always predicted as lower than the real values. It should be mentioned that the mean absolute percentage of error with the BAM model is considerably lower than other models in this case. The BAM model can predict shear stress much more accurately than other models even in this case. As seen in Table 1, the statistical parameter values of the BAM and ZAM models are significantly smaller than other geometrical methods. However, the ZAM model produced good results, but it requires solving many equations for estimating shear stress distribution. On the other hand, the BAM method can estimate shear stress distribution in compound channel sub-sections with high precision and uses simpler and fewer relations than ZAM. It can be deducted that the BAM method is robust in predicting the shear stress distribution along the wetted perimeter of compound channels.

\section{Conclusion}

The present study expressed five geometrical and analytical models to predict the shear stress distribution in compound channels. These models were compared with experimental data, and the ability of each model in predicting the shear stress in sub-sections of a compound channel was investigated. In four models, the effect of secondary flows on predicting shear stress distribution was ignored, whereas this effect was considered only in ZAM. Based on the obtained results, YLM and KPM predicted the same pattern of shear stress distribution for a floodplain wall and main channel wall, where the shear stress values increased with increasing wetted perimeter. YLM and KPM exhibited poor performance in estimating the shear stress distribution on compound channel walls with average RMSE of 0.49 and 0.53 , respectively. According to the results, KPM produced adequate results in estimating the shear stress distribution on a floodplain bed with low flow depth. However, with increasing flow depth, the results deviated from the laboratorial outcomes. In fact, KPM estimated the shear stress distribution on a floodplain bed and main channel bed better than YLM. However, both methods were much weaker in predicting shear stress distribution than the other mentioned methods. The SKM estimations of shear stress distribution for the wetted perimeter of a wall and bed of a floodplain followed a straight line and were close to experimental data for a floodplain wall. For a floodplain bed far from the corners, the SKM results were compatible with laboratorial data. The pattern of shear stress distribution on the wall and bed of a main channel estimated by SKM was more acceptable and the results were better than YLM and KPM. The BAM model performed well in predicting floodplain and main channel shear stress, and it can be deducted that the results for all flow depths are compatible with laboratorial outcomes. ZAM presented smaller error percentages than the geometric methods pointed out. The ZAM predictions for floodplain and main channel walls at the beginning of the wetted perimeter were much lower than experimental data, but after the beginning point, the estimations were closer to observed data. From all models, the effect of secondary flow was only considered in ZAM, which resulted in a superior performance to other models in predicting shear stress in compound channels. Among the methods in which the effect of secondary flow was ignored, BAM performed most appropriately. It is deducted that both BAM and ZAM are more accurate than other models, with mean RMSE of 0.058, and 0.049 , respectively. It is noted that in the BAM model, solving implicit equations is required to compute the Lagrange multipliers. On the other hand, ZAM calls for solving several equations for predicting shear stress in compound channels, it is time-consuming and requires high accuracy. In this regard, the BAM model necessitates fewer equations. Therefore, BAM may be selected as the most suitable model for estimating the shear stress distribution in compound channels among the models presented in this study. The authors of the present study investigated a novel, simpler equation to avoid solving implicit equations in BAM for predicting shear stress distribution with high precision.

\section{References}

1. Knight, D.W., Yuen, K.W.H. and Al Hamid, A.A.I. "Boundary shear stress distributions in open channel flow", In: K. Beven, P. Chatwin, and J. Millbank (eds), Physical Mechanisms of Mixing and Transport in the Environment, Wiley New York, pp. 51-87 (1994).

2. Prasad, B.V.R. and Manson, J.R. "Discussion of a geometrical method for computing the distribution of boundary shear stress across irregular straight open channels", J. Hydraul. Res., 40(4), pp. 537-539 (2002).

3. Guo, J. and Julien, P.Y. "Shear stress in smooth rectangular open-channel flow", J. Hydraul. Eng., 131(1), pp. 30-37 (2005).

4. Lane, E.W. "Progress report on studies on the design 
of stable channels by the bureau of reclamation", $J$. Hydraul. Div., 79(280), pp. 1-30 (1953).

5. Cruf, R.W. "Cross section transfer of linear momentum in smooth rectangular channel", Geological Survey Water Supply paper 1592-B, U.S. Government Printing Office, Washington D.C. (1965).

6. Myers, W.R.C. "Momentum transfer in a compound channel", J. Hydraul. Res., 16(2), pp. 139-150 (1978).

7. Yuen, K.W.H. "A study of boundary shear stress, flow resistance and momentum transfer in open channels with simple and trapezoidal cross section", PhD Thesis, The University of Birmingham (1989).

8. Knight, D.W. and Sterling, M. "Boundary shear in circular pipes partially full", J. Hydraul. Eng., 126(4), pp. 263-275 (2000).

9. Seckin, G., Seckin, N. and Yurtal, R. "Boundary shear stress analysis in smooth rectangular channels", Can. J. Civil Eng., 33, pp. 336-342 (2006).

10. Yang, S.Q. and Lim, S.Y. "Mechanism of energy transportation and turbulent flow in a 3D channel", J. Hydraul. Eng., 123(8), pp. 684-692 (1997).

11. Khodashenas, S.R. and Paquier, A. "A geometrical method for computing the distribution of boundary shear stress across irregular straight open channel", $J$. Hydraul. Res., 37, pp. 381-388 (1999).

12. Sterling, M. and Knight, D.W. "An attempt at using the entropy approach to predict the transverse distribution of boundary shear stress in open channel flow", Stoch. Env. Res. Risk Assess., 16, pp. 127-142 (2002).

13. Berlamont, J.E., Trouw, K. and Luyckx, G. "Shear stress distribution in partially filled pipes", J. Hydraul. Eng., 129(9), pp. 697-705 (2003).

14. Yang, S.Q. and Lim, S.Y. "Boundary shear stress distribution in trapezoidal channels", J. Hydraul. Res., 43(1), pp. 98-102 (2005).

15. Bonakdari, H., Larrarte, F. and Joannis, C. "Study of shear stress in narrow channels: application to sewers", Urban Water, 5(1), pp. 15-20 (2008).

16. El Kadi Abderrezzak, K. "Evolution of the riverbed due to flow and sediment transport", PhD Thesis, University of Claude Bernard, Lyon (2006).

17. Javid, S. and Mohammadi, M. "Boundary shear stress in a trapezoidal channel", Int. J. Eng., 25(4), pp. 365373 (2012).

18. Kabiri-Samani, A., Farshi, F. and Chamani, M.R. "Boundary shear stress in smooth trapezoidal open channel flows", J. Hydraul. Eng., 139(2), pp. 205-212 (2013).

19. Bonakdari, H., Sheikh, Z. and Tooshmalani, M. "Comparison between Shannon and Tsallis entropies for prediction of shear stress distribution in circular open channels", Stoch. Env. Res. Risk Assess., 29(1), pp. 1-11 (2015).

20. Bonakdari, H., Tooshmalani, M. and Sheikh, Z. "Predicting shear stress distribution in rectangular channels using entropy concept", Int. J. Eng., 28(3), pp. 360-367 (2015).
21. Sheikh, Z. and Bonakdari, H. "Prediction of boundary shear stress in circular and trapezoidal channels with entropy concept", Urban Water, 13(6), pp. 629-636 (2015).

22. Chiu, C.L. "Application of entropy concept in open channel flows", J. Hydraul. Eng., 117(5), pp. 615-628 (1991).

23. Araujo, J.C. and Chaudhry, F.H. "Experimental evaluation of 2-D entropy model for open channel flow", $J$. Hydraul. Eng., 124(10), pp. 1064-1067 (1998).

24. Zarrati, A.R., Jin, Y.C. and Karimpour, S. "Semianalytical model for shear stress distribution in simple and compound open channels", J. Hydraul. Eng., 134(2), pp. 205-215 (2008).

25. Rajaratnam, N. and Ahmadi, R. "Hydraulics of channels with flood-plains", J. Hydraul. Res., 19(1), pp. 43-60 (1981).

26. Myers, W.R.C. and Elsawy, E.M. "Boundary shear in channel with flood-plain", J. Hydraul. Div., 101(7), pp. 933-946 (1975).

27. Cokljat, D. and Younis, B.A. "Second-order closure study of open-channel flows", J. Hydraul. Eng., 121(2), pp. 94-107 (1995).

\section{Biographies}

Zohreh Sheikh Khozani is now $\mathrm{PhD}$ candidate in Hydraulic structures (Civil Engineering), Faculty of Engineering, University of Razi, Kermanshah, Iran. She obtained MSc degree from Semnan University in 2011. She has 6 published papers in ISI journals and more than 4 conference presentations. She works in field of shear stress distribution, sediment transport in open channels and rivers, and use of entropy concept and soft computing methods in engineering applications.

Hossein Bonakdari is a Professor, Department of Civil Engineering of University of Razi, earned his $\mathrm{PhD}$ in Civil Engineering in the University of CaenFrance. He has spent his MSc in civil engineering, hydraulics structures in University of Ferdowsi, Mashhad, Iran. His fields of specialization and interest include metrology and modeling of wastewater urban drainage systems, soft computing, sediment transport, computational fluid dynamic and hydraulics, design of hydraulic structures, and fluid mechanics. From 2010 till 2011, he has been a researcher at Laboratory of Civil and Environmental Engineering, INSA of Lyon, France. Results obtained from his researches have been published in more than 65 papers in international journals (h-index=8). He has also more than 150 presentations in national and international conferences. He published two books. 